\title{
Editorial: Special Issue on Recent Advances in Cognitive Learning and Data Analysis
}

\author{
Jinchang Ren ${ }^{1} \cdot$ Amir Hussain $^{2} \cdot$ Jiangbin Zheng ${ }^{3} \cdot$ Cheng-Lin Liu ${ }^{4} \cdot$ Bin Luo $^{5}$ \\ Published online: 15 June 2020 \\ (C) Springer Science+Business Media, LLC, part of Springer Nature 2020
}

With the rapid development of artificial intelligence (AI)-related techniques and the continuous explosion of multimodal data, there is a new trend in combining AI machine learning with multimodal big data analytics. This has enabled emerging new techniques and applications in a wide range of fields. Accordingly, these have brought in both challenges in effective big data analysis and opportunities in innovative applications. To this end, cognitive modeling and cognitive systems have attracted increasing attention under the framework of big data enabled machine learning, especially the sparse representation and sparse learning, deep learning, and reinforcement learning.

To address these challenges and opportunities, we have successfully organized the Brain Inspired Cognitive System (BICS) Conference series, including the $9^{\text {th }}$ in Xi' an, China in July 2018 [1] and $10^{\text {th }}$ in Guangzhou, China in July 2019 [2]. Selected papers are extended and included in this special issue. The special issue has solicited the state-of-the-art

Jinchang Ren

jinchang.ren@strath.ac.uk

Amir Hussain

Hussain.doctor@gmail.com

Jiangbin Zheng

zhengjb@nwpu.edu.cn

Cheng-Lin Liu

liucl@nlpr.ia.ac.cn

Bin Luo

luobin@ahu.edu.cn

1 Department of Electronic and Electrical Engineering, University of Strathclyde, Glasgow, UK

2 School of Computing, Edinburgh Napier University, Edinburgh, UK

3 School of Software, Northwestern Polytechnical University, Xi'an, China

4 Institute of Automation, Chinese Academy of Sciences, Beijing, China

5 School of Computer Science and Technology, Anhui University, Hefei, China contributions in cognitive learning and data analysis, which has also provided a premier forum for both the academic and industrial research community to report progress, exchange findings, and facilitate future multidisciplinary research directions as detailed below.

In total, there are seven papers included in this special issue. The selected papers have covered a wide range of relevant topics, showing both theoretical and applicable values. Therefore, these can be categorized into various groups under different criteria, where detailed introduction of the included papers is given as follows.

\section{From Conventional Machine Learning to Deep Learning}

In the seven contributed papers, three of them papers are deep learning based, including Style Neutralization Generative Adversarial Network (SN-GAN) upgraded U-Net [3], SemiSupervised Convolutional Neural Network [4], and recurrent neural network (RNN) [5]. On the other hand, conventional machine learning approaches are also adopted in the other four papers, which include multi-scale mahalanobis kernel-based support vector machine [6], graph model based salient superpixel visual tracking [7], an evolutionary safelevel synthetic minority over-sampling technique (ESLSMOTE) for balanced learning [8], and Laplacian-regularized correlative sparse ranking enabed matching [9]. The transition from conventional approaches to deep learning has shown a changing trend in $\mathrm{AI}$ and cognitive computing.

\section{Various Tasks}

The seven contributed papers in the special issue cover several typical tasks of cognitive computation. These include classification/recognition [3, 4, 6, 9], image captioning [5], and object segmentation for visual tracking [7]. The work in [8] is also tested on three classifiers, where the work in [9] for 
vehicle re-identification can also be regarded as classification or recognition. Majority of the papers focus on pattern classification and recognition, which has indicated this as the mainstream of cognitive computation.

\section{Diverse Application Areas}

The application areas of the seven contributed papers are quite diverse. These cover from data classification [3], including seminal quality assessment [8], to synthetic aperture radar image recognition [4], image captioning [5], high-resolution remote sensing image classification [6], visual tracking [7], and vehicle re-identification [9]. These have demonstrated the growing wider application areas of cognitive computation.

In addition, the SN-GAN model [3] and the ESLSMOTE approach [8] can actually be applied in more generic applications, as these address mainly on theoretical side of machine learning, an important topic in cognitive computation. The remaining approaches focus on application sides, where improved models and methods are adapted to particular problems and show promising results.

In summary, this special issue has covered several challenging topics within cognitive computation. All these have been valuable and useful in AI and cognitive analysis of data and patterns from various application areas. Herein we in particular thank the great contributions from all authors, the efforts from anonymous reviewers, and the management and Editorial team of the Cognitive Computation Journal.

\section{References}

1. Ren J, Hussain A, Zheng J, Liu C-L, Luo B, Zhao H, Zhao X, et al. (eds). Advances in brain-inspired cognitive systems: 9th International Conference, BICS 2018, Xi'an, China, July 7-8, 2018, Proceedings. Cham: Springer, 2018. 850 p. (Lecture Notes in Artificial Intelligence). https://doi.org/10.1007/978-3-030-00563-4

2. Ren J, Hussain A, Zhao H, Huang K, Zheng J, Cai J, Chen R, Xiao Y, et al. (eds). Advances in brain inspired cognitive systems - 10th International Conference, BICS 2019, Guangzhou, China, July 1314, 2019, Proceedings. Lecture Notes in Computer Science 11691, Springer 2020.

3. Jiang H, Huang K, Zhang R, et al. Style-neutralized pattern classification based on adversarially trained upgraded U-net. Cogn Comput. 2019. https://doi.org/10.1007/s12559-019-09660-0

4. Yue Z, Gao F, Xiong Q, et al. A novel semi-supervised convolutional neural network method for synthetic aperture radar image recognition. Cogn Comput. 2019. https://doi.org/10.1007/s12559-019-09639-x

5. Chen H, Ding G, Lin Z, et al. Image captioning with memorized knowledge. Cogn Comput. 2019. https://doi.org/10.1007/s12559019-09656-w

6. Sun G, Rong X, Zhang A, et al. Multi-scale mahalanobis kernelbased support vector machine for classification of high-resolution remote sensing images. Cogn Comput. 2019. https://doi.org/10. 1007/s12559-019-09631-5

7. Zhan J, Zhao H, Zheng P, et al. Salient superpixel visual tracking with graph model and iterative segmentation. Cogn Comput. 2019. https://doi.org/10.1007/s12559-019-09662-y

8. Ma J, Afolabi DO, Ren J, et al. Predicting seminal quality via imbalanced learning with evolutionary safe-level synthetic minority oversampling technique. Cogn Comput. 2019. https://doi.org/10.1007/ s12559-019-09657-9

9. Zheng A, Dong J, Lin X, et al. Visual cognition-inspired multi-view vehicle re-identification via laplacian-regularized correlative sparse ranking. Cogn Comput. 2019. https://doi.org/10.1007/s12559-019-09687-3

Publisher's Note Springer Nature remains neutral with regard to jurisdictional claims in published maps and institutional affiliations. 